\title{
COTS based GNSS Receiver with Precise Point Positioning for CubeSats
}

\author{
Alexandru Pandele ${ }^{1, *}$, Costel Cherciu ${ }^{1}$, Marius Trușculescu, Claudiu Drăgășanu ${ }^{1}$ and \\ Sergiu-Ştefan Mihai ${ }^{1}$ \\ ${ }^{1}$ Romanian InSpace Engineering SRL, Bucharest, Romania
}

\begin{abstract}
The paper presents the work towards developing a COTS based GNSS receiver and integrating Precise Point Positioning algorithms to facilitate close proximity operations for CubeSats in formation flying and during docking or rendezvous manoeuvres. We initially present the driving requirements identified for these types of missions. Besides fitting a standard CubeSat, the receiver has to weigh less than $0.3 \mathrm{~kg}$, consume less than $5 \mathrm{~W}$ and be multi-frequency and multi-constellation. Next, follows the identification of a commercial off the shelf GNSS receiver that can be easily customized to fit the basic requirements of a GNSS space receiver and on which Precise Orbit Determination (POD) algorithms can be implemented. For the start of the activity three commercial receivers were selected as proposed candidates to be traded off regarding the degree to which they fulfil the requirements, the degree of openness and of manufacturer support. A COTS microcontroller shall be then selected to control the operation of the COTS receiver. We then expand on the proposed general architecture of the system from COTS modules to their integration philosophy, with a discussion on the means of delivering the correction factors to the receiver. PPP corrections are expected to be delivered either via ground stations or via the geostationary satellite based commercial services. The PPP algorithms are to be implemented on the microcontroller, which will also try to maximize the availability of a precise PVT solution by incorporating a neural network fed by an orbit propagator and the PPP algorithm. The neural network shall estimate a precise position whenever PPP corrections are not available. The training of the neural network shall be done on the ground, allowing for a small footprint on board. A preliminary design of the hardware and the planned qualification plan is concluding the work.
\end{abstract}

\section{Introduction}

The American Global Positioning System (GPS) started to be deployed in 1989. In 1992, when the constellation was still under deployment (deployment finished in 1995), the first space mission that made use of the new technology was already launched. Topex/Poseidon, a combined US/French mission, featured a $28 \mathrm{~kg}$ GPS payload with 6 channels, aiming to characterize the atmosphere using the radio occultation of GPS signals[1]. Other purposes of

\footnotetext{
${ }^{*}$ Corresponding author: alexandru.pandele@roinspace.com
} 
using GNSS receivers in space are positioning, orbit determination, timing, constellation control and formation flying. This usage for GNSS receivers is interesting also in the small satellites market, where Frost \& Sullivan experts projected impressive developments in the next years [2]:

- Total projected launch capacity: $\mathbf{1 1 , 7 4 6}$ small satellites;

- Total payload mass of $\mathbf{2 , 7 5 8}$ tonnes of small satellites;

- Cumulative percentage of 0 - $15 \mathrm{Kg}$ and $150-500 \mathrm{Kg}-\mathbf{7 3 . 8 \%}$ of the small-satellite launch demand;

- $\quad \mathbf{9 7 . 7 \%}$ of the total payload launch mass demand will be generated by commercial operators, with the major contributors being Space X, EarthNow, and Oneweb;

- $\quad 37$ small-satellite commercial operators will generate more than $\mathbf{9 0 \%}$ of the launch demand for their constellation installation and replacement missions.

Considering this expert estimation of the small satellite market in the next decade and our experience with integrating COTS components for space usage, RISE is aiming to develop OrbFIX, a low cost GNSS receiver able to provide lower than $10 \mathrm{~cm}$ accuracy via PPP and making use of multiple GNSS constellations and frequencies.

\section{Technical approach}

\subsection{Requirements definition}

The first step in the development of the OrbFIX receiver is the definition of a set of requirements. Given the targeted market, the following preliminary requirements emerged. The $300 \mathrm{~g}$ mass limit is less than $1 / 3$ of the 1 Unit Cubesat. The module should allow several operation modes, each of them dedicated to a specific maximum and average power consumption. The temperature range is specific to the LEO environment. The supply voltage is also standard for cubesat platforms, as well es the communication with the on-board computer. It should allow at least the usage of GPS and Galileo constellations on both main frequencies, L1 and L5. The PPP correction source is critical to achieving high performance positioning. The OrbFIX basic requirements are summarized in

Table 1. OrbFIX basic requirements.

\begin{tabular}{|l|l|}
\hline Subject of the requirement & Requirement \\
\hline Volume & $<1$ Unit CubeSat \\
\hline Mass & $<=300 \mathrm{~g}$ \\
\hline Maximum power & $<=4 \mathrm{~W}$ \\
\hline Average power & $<=2 \mathrm{~W}$ \\
\hline Operational temperature & {$[-30,+60]^{\circ} \mathrm{C}$} \\
\hline Non-operational temperature & {$[-40,+70]^{\circ} \mathrm{C}$} \\
\hline Supply voltage & $3 \mathrm{~V} 3$ and $/$ or $5 \mathrm{~V}$ \\
\hline Communication & $\mathrm{CAN} / \mathrm{I} 2 \mathrm{C} / \mathrm{SPI}$ \\
\hline Constellations & $\mathrm{GPS} \&$ GALILEO \\
\hline Frequencies & $\mathrm{L} 1 / \mathrm{E} 1$ \& L5/E5a \\
\hline PPP correction source & $\begin{array}{l}\text { satellite and/or ground } \\
\text { station radio connection }\end{array}$ \\
\hline Tracked satellites & $>=24$ \\
\hline
\end{tabular}

\subsection{Architecture}


As already stated, OrbFIX shall be based on COTS components. It is built around a COTS GNSS receiver that is already fulfilling the main requirements as they were stated before. A COTS antenna shall be used to provide the input signal to the receiver, whereas an MCU, that can be upgraded to a radiation hardened version, shall take care of the communication with the satellite BUS. The MCU shall implement various modules, such as the PPP module responsible for the determination of the precise position, a precise orbit determination algorithm that shall compute the satellite orbit based on the precise positions, a light orbit propagator that should act as a last low power positioning module and a neural network that shall try to improve the orbit propagator positions with timely GNSS initializations. The current architecture shall include Single Event Effect protection in order to increase the availability of the receiver.

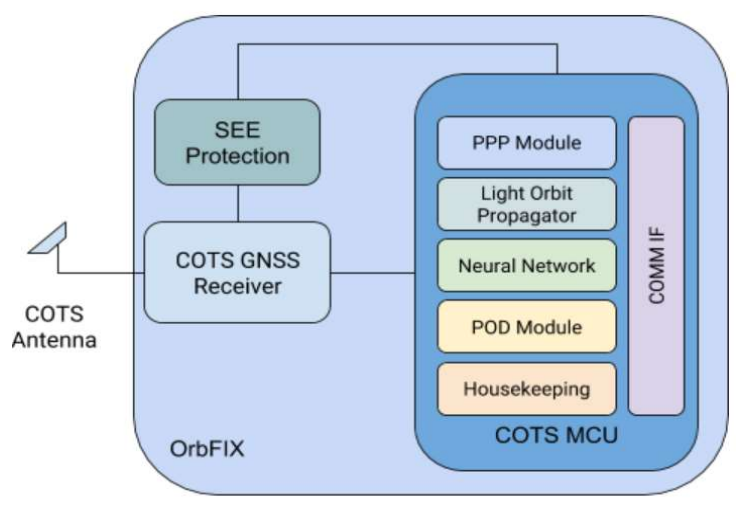

Fig. 1. OrbFIX block architecture.

\subsection{Precise Point Positioning}

GNSS clocks and orbits are broadcasted in the navigation message of each satellite. However, due to various reasons, including the low datarate of the GNSS message transmission, these parameters include errors in the order of several meters, as it can be seen in Fig. 2 for GPS (left) and GALILEO (right).
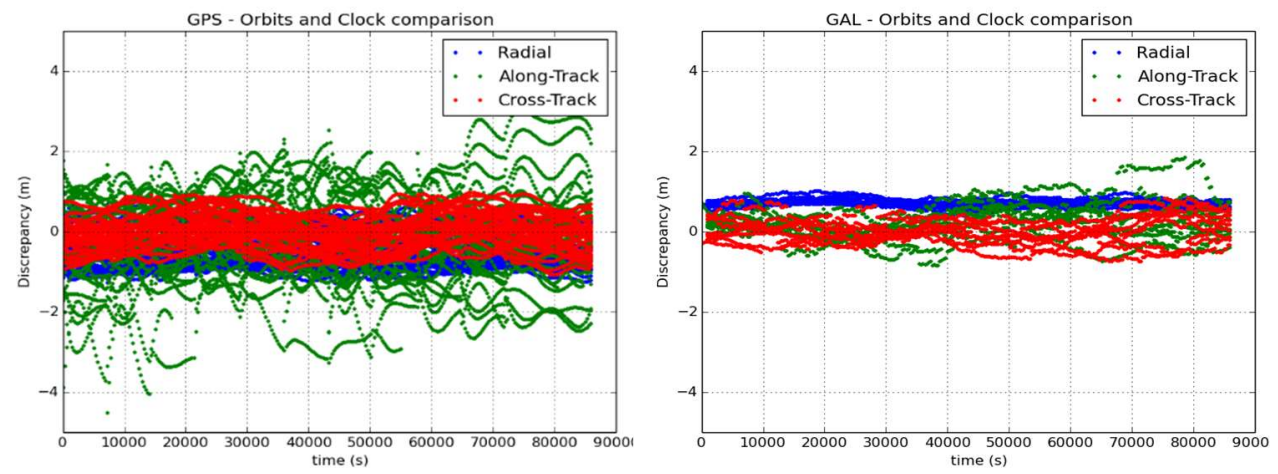

Fig. 2. GPS and GALILEO orbits and clocks errors [3]

Correcting orbits, clocks and ionospheric parameters PPP can reduce the positioning error to centimetre level. In Fig. 3, on the right, it can be seen the "regular" positioning error, using 
the GRAPHIC combination between the GNSS code and carrier. On the left, the same receiver data is corrected with precise orbits and clocks in a PPP algorithm. The error is reduced by more than $50 \%$. It is to be noted that the data used for these figures comes from a single frequency GPS receiver on-board GRACE mission. The results are expected to improve further when using multiple constellations and frequencies.
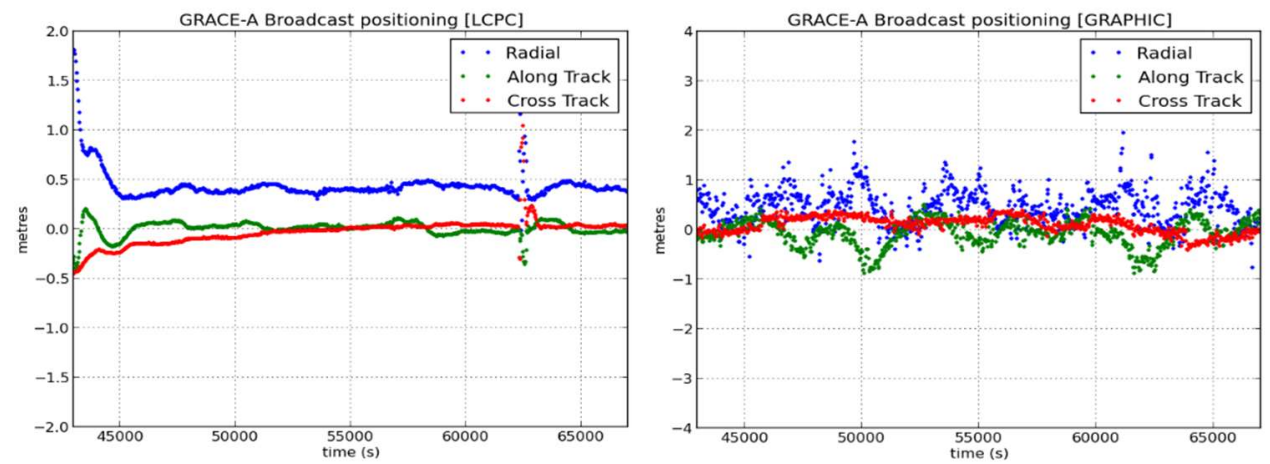

Fig. 3. PPP and GRAPHIC corrected positioning errors for GRACE-A satellite [3]

The OrbFIX in orbit demonstrator shall use only ground stations to provide PPP corrections to the satellite. However, several additional options are being explored for this service. One makes use of the commercial services already available on the market where PPP corrections are being broadcasted via Geostationary satellites. Another one could use commercial communications constellations such as OneWeb, Starlink or Globalstar. A third option is the planned GALILEO High Accuracy Service that is going to be distributed by GALILEO satellites via the E6 frequency.

The target market of OrbFIX consists of small and nanosatellites. The latter suffer in general from low power budgets, as the solar panel real estate is very limited for them. While OrbFIX uses less than $2 \mathrm{~W}$ in full feature, PPP mode, it offers also a large flexibility from the power consumption point of view, by providing several low-power operation modes. A tradeoff on performance is made in this case. The three low power modes are:

- $<70 \mathrm{~mW}$ (Lowest power consumption): light orbit propagator $(<100 \mathrm{~m}$ positioning error after one orbit)

- $\quad<200 \mathrm{~mW}$ : orbit propagator with GNSS reinitialization every 10 minutes $(<50$ $\mathrm{m}$ positioning error, aiming to $<20 \mathrm{~m}$ with $\mathrm{ANN}$ )

- $\quad<800 \mathrm{~mW}$ : orbit propagator + GNSS positioning $(<10 \mathrm{~m})$

The Light Orbit Propagator can, as previously mentioned, give a good estimation for the position. More precise results can be achieved using a trained Artificial Neural Network that learns and compensates the errors which rapidly accumulate over time. The ANN shall also receive information from the GNSS measurements, in order to better improve the estimated position. The GNSS is queried at specific time intervals, in order to efficiently use the power budget. 


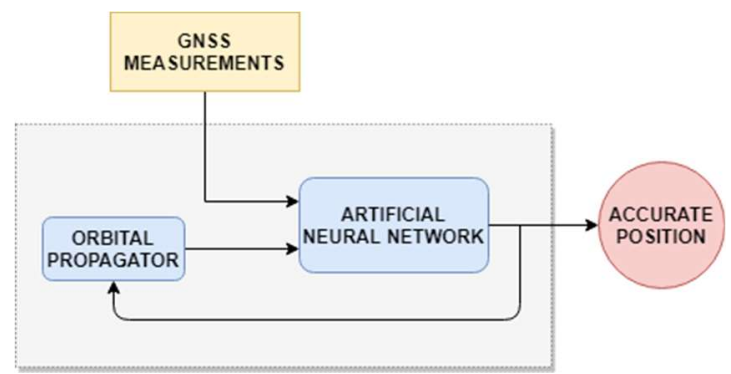

Fig. 4. Artificial Neural Network usage for improved satellite position

\section{Conclusions}

Answering to growing market requirements for small and nano-satellites, RISE is proposing the development of a Precise Point Positioning GNSS receiver based on Components Off the Shelf. This article summarized the justification and the technical approach for such hardware.

\section{References}

1. ***JPL/NASA Topex/Poseidon Mission webpage: https://www.jpl.nasa.gov/missions/topex-poseidon/

2. *** Small Satellite Launch Market Revenues To Exceed 69 Billion By 2030 Report, spacewatch.global, https://spacewatch.global/2019/01/small-satellite-launch-marketrevenues-to-exceed-69-billion-by-2030-report/

3. J.Sanz Subirana et al., GNSS Data Processing (ESA Communications, 2013) 